\title{
Beyond antigens and adjuvants: formulating future vaccines
}

\author{
Tyson J. Moyer, ${ }^{1,2}$ Andrew C. Zmolek, ${ }^{1,2,3}$ and Darrell J. Irvine ${ }^{1,2,4,5,6,7}$ \\ ${ }^{1}$ Center for HIV/AIDS Vaccine Immunology and Immunogen Discovery, ${ }^{2}$ David H. Koch Institute for Integrative Cancer Research, ${ }^{3}$ Department of Chemical Engineering, ${ }^{4}$ Department of Materials Science \\ and Engineering, and ${ }^{5}$ Department of Biological Engineering, Massachusetts Institute of Technology, Cambridge, Massachusetts, USA. ${ }^{6}$ Ragon Institute of MCH, MIT and Harvard, \\ Cambridge, Massachusetts, USA. ${ }^{7}$ Howard Hughes Medical Institute, Chevy Chase, Maryland, USA
}

\begin{abstract}
The need to optimize vaccine potency while minimizing toxicity in healthy recipients has motivated studies of the formulation of vaccines to control how, when, and where antigens and adjuvants encounter immune cells and other cells/tissues following administration. An effective subunit vaccine must traffic to lymph nodes (LNs), activate both the innate and adaptive arms of the immune system, and persist for a sufficient time to promote a mature immune response. Here, we review approaches to tailor these three aspects of vaccine function through optimized formulations. Traditional vaccine adjuvants activate innate immune cells, promote cell-mediated transport of antigen to lymphoid tissues, and promote antigen retention in LNs. Recent studies using nanoparticles and other lymphatic-targeting strategies suggest that direct targeting of antigens and adjuvant compounds to LNs can also enhance vaccine potency without sacrificing safety. The use of formulations to regulate biodistribution and promote antigen and inflammatory cue co-uptake in immune cells may be important for next-generation molecular adjuvants. Finally, strategies to program vaccine kinetics through novel formulation and delivery strategies provide another means to enhance immune responses independent of the choice of adjuvant. These technologies offer the prospect of enhanced efficacy while maintaining high safety profiles necessary for successful vaccines.
\end{abstract}

\section{Introduction}

Vaccines are a proven, powerful tool for public health and have had a major effect on medicine and society. Vaccine development has been very successful, with 81 currently FDA-licensed products in the US and many more worldwide. However, this success has had a natural sieving effect, removing the easier to neutralize microbes from the list of remaining challenges, and many of the important pathogens for which no effective vaccine exists (e.g., malaria, HIV, tuberculosis, various bacteria) present daunting immunological obstacles (1-3). In addition to these challenges, modern standards for vaccine safety, in which adverse events at frequencies comparable to those of rare genetic disorders in the population are viewed as unacceptable, add to the challenge of solving the vaccine puzzle for new pathogen threats. These challenges fuel interest in new technologies that can help shape the strength and quality of immune responses to vaccination and provide new means to induce potent immune responses with a high degree of safety.

Vaccines typically comprise an antigen (the target for the immune response) and an adjuvant (a coadministered compound meant to promote the immune response to the antigen); vaccines based on defined target antigens are known as subunit vaccines (4). In the last 15 years, much effort in vaccinology has focused on

Authorship note: T.J. Moyer and A.C. Zmolek contributed equally to this work and serve as co-first authors.

Conflict of interest: D.J. Irvine is a consultant and equity holder in Vedantra Pharmaceuticals and Torque Therapeutics Inc. and holds patents related to vaccines and immunotherapy technologies.

Reference information: J Clin Invest. 2016;126(3):799-808. doi:10.1172/JCI81083. the development of adjuvants based on defined "danger signals," conserved molecular motifs signifying the presence of dangerous microbes that are recognized by specific pattern recognition receptors (PRRs) in host stromal or immune cells (5). In the case of live-attenuated vaccines, the weakened pathogen is both antigen and adjuvant due to the endogenous danger signals (e.g., viral or bacterial nucleic acids) present within the microbe itself. However, in many current vaccine candidates, the antigen is a welldefined recombinant protein, and the adjuvant is composed of one or more molecular danger signals, such as monophosphoryl lipid A, CpG DNA, or polyinosinic:polycytidylic acid (6). Such molecular vaccines, like any other drug from the broader field of pharmaceuticals, can require formulation technologies that ensure that the biodistribution and pharmacokinetics of the vaccine are optimized for potency and safety. Therefore, many current "antigen plus adjuvant" vaccine concepts are better defined as comprising three components - antigen, adjuvant, and vaccine formulation.

Several outstanding fundamental problems are relevant for producing new subunit vaccines against infectious disease and tumors. Prophylactic vaccines often fail to elicit durable protection through the production of sufficient long-lived antibody-producing plasma cells and memory $\mathrm{T}$ and $\mathrm{B}$ cell populations $(7,8)$; methods to promote sustained immunity are needed. Broadly neutralizing antibodies against some microbes, such as HIV, appear to require relatively high levels of somatic hypermutation (SHM) (9), the process by which antibodies are mutated away from their germline sequence to refine their specificity and affinity for target antigens. Methods to promote germinal center (GC) reactions in which SHM occurs are a focus of ongoing research. For therapeutic vaccines against cancer and intracellular microbes, strong 


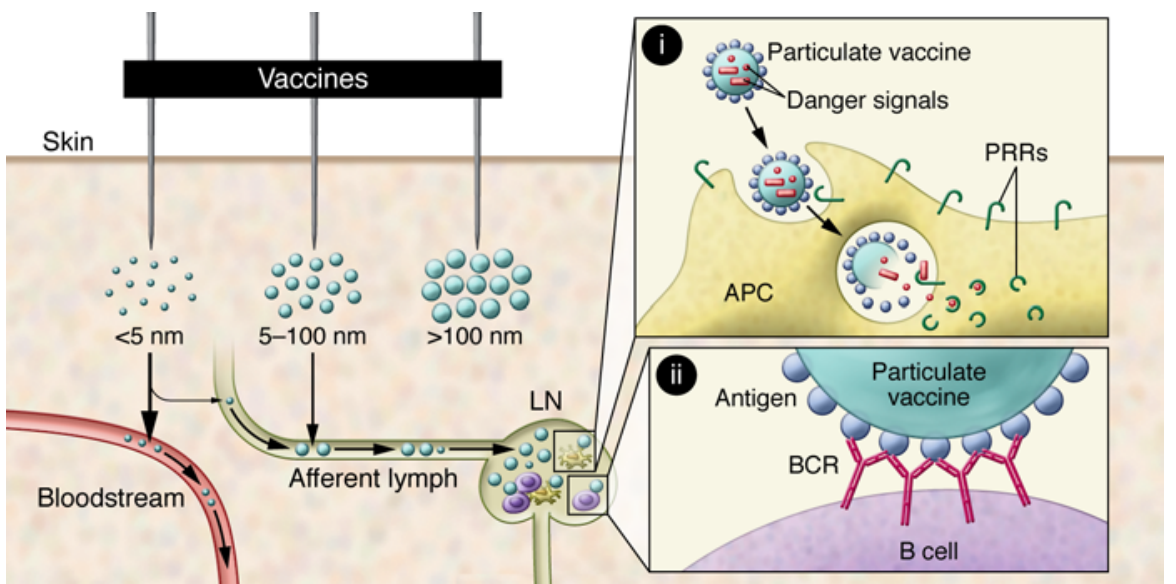

\begin{abstract}
Figure 1. Roles of particulate vaccine formulations in controlling vaccine biodistribution and presentation to immune cells. Particulate vaccines in the 20 - to $100-\mathrm{nm}$ size range preferentially traffic into lymphatic vessels towards the LNs, while smaller molecules disseminate into the systemic circulation with poor lymphatic uptake. Larger particles become trapped in tissue and tend to depot near the site of injection. (i) Particulate vaccines containing both antigen and danger signals can codeliver these vaccine components to the same APC, enabling activation of PRRs selectively in cells that have acquired antigen. (ii) A high-density display of antigen on the surface of particulate vaccines enhances cross-linking of antigen receptors on specific B cells.
\end{abstract}

$\mathrm{CD}^{+} \mathrm{T}$ cell responses are required, and methods to elicit strong $\mathrm{T}$ cell immunity in humans using safe, non-live vaccines remain an ongoing challenge. Finally, the use of adjuvants to enable subunit vaccines to approach the potency of live infections introduces serious safety concerns, and strategies to safely use powerful danger signals in vaccination will likely be important for tackling several of the issues described above.

In this brief Review, we highlight recent efforts in vaccine formulation that aim to address some of these immunological challenges, focusing in particular on transport of vaccines to lymphoid tissues, delivery of danger signals to immune cells, and using materials to regulate the kinetics of subunit vaccine presentation to immune cells. Key issues are the distribution of adjuvant compounds into lymphoid tissues versus the systemic circulation and codelivery of antigen and adjuvant to the same cells. In addition, because the immune system has evolved to intrinsically recognize the particulate state of microbes (viruses, typically nanoparticles; bacteria, typically microparticles), the design of subunit vaccines that "rebuild" selected features of microbial structure, such as the display of antigen at the surface of particles or the repetitive display of molecular danger signals, has been shown to significantly affect the immune response. Finally, the timing with which the immune system is exposed to antigen and inflammatory cues is known to play a critical role in the immune response to infectious agents but remains an understudied area in synthetic vaccines. Vaccine delivery approaches that could tailor the kinetics of vaccine uptake in lymphoid tissues thus provide another means to shape the resulting immune response. Addressing these issues requires a combination of efforts in pharmaceutical sciences, materials science, medicinal chemistry, and protein engineering.

\section{Controlling vaccine biodistribution}

Cell-mediated versus lymphatic transport for vaccine delivery to lymph nodes. Naive T cells and B cells recirculate through the lymph nodes (LNs) and spleen, and thus vaccines must physically access these organs to elicit an immune response. Trafficking of vaccines from an injection site to LNs can be mediated by lymphatic drainage or through immune cell-mediated transport. "First-generation" adjuvants, such as aluminum salts (alum) and MF59 (an oil-in-water nanoemulsion), function by promoting antigen-presenting cell (APC) migration to intramuscular injection sites, in which vaccine antigen is internalized and then transported by neutrophils, monocytes, and DCs to draining LNs to support T cell responses (10). Antigen also drains from the intramuscular injection site, as MF59 does not directly associate with antigens (11) and not all antigen is retained at the injection site with alum (12); MF59 can also promote antigen retention in LNs that likely supports B cell responses (13). The intramuscular route of administration has long been favored because these adjuvants can elicit significant injection site reactions when administered at other sites (e.g., subcutaneous), and, for many vaccines, intramuscular administration with alum or oilin-water emulsions promotes robust, protective humoral responses in humans. However, APCs trafficking vaccine to LNs have been shown in most instances to represent a small population (e.g., $\sim 1 \%-5 \%$ of all DCs in a LN); thus, a minute quantity of antigen is typically detectable in muscle-draining LNs with traditional adjuvants $(10,11)$, which may limit the strength of the immune response in ways that could be important for intracellular pathogens. An alternative is to use subcutaneous or intradermal routes of injection where lymphatic drainage is efficient to increase direct vaccine delivery to LNs, combined with formulation strategies that eliminate or minimize injection site reactions. A number of approaches now exist that enable vaccines to accumulate at high levels in LNs from these sites, reaching a majority of DCs in a node.

For direct lymphatic drainage, the molecular weight and dimensions of vaccine particles are critical because fluid clearance from peripheral tissues is gated by physical size: the blood clears 10-fold more fluid from tissues than lymph, but transport across the basement membrane and between tight junctions of endothelial cells is highly inefficient for large macromolecules. Thus, there exists a nearly linear dependence of lymphatic uptake and molecular weight, up to a plateau value of approximately $45 \mathrm{kDa}$ in size, above which proteins show nearly $100 \%$ trafficking to lymph in both animal models and humans (refs. 14, 15, and Figure 1). This relationship between molecular size and lymph uptake explains why low-molecular-weight adjuvants like imidazoquinolines show high levels of systemic exposure/inflammatory toxicity following parenteral injection (16) and partly explains the poor potency of low-molecular-weight antigens like peptides. Efficient trafficking to lymphoid tissues is only a first step; vaccine materials must also be subsequently captured in LNs. Material that flushes through the LN will eventually be delivered to the systemic circulation through 
the thoracic duct, providing a second route for systemic exposure to antigens and adjuvants. That such systemic dissemination is possible is illustrated by studies with small (30 $\mathrm{nm}$ in diameter) PEGylated polymer particles, which, following intradermal injection in mice, were shown to reach concentrations in the blood comparable to a direct i.v. injection after 18 hours (17).

The size-based regulation of lymph versus blood trafficking described above has motivated the exploration of nanoparticles as carriers for vaccine delivery to LNs $(18,19)$. The efficiency of direct lymphatic trafficking of nanoparticles is hindered by convection through the extracellular matrix (ECM) as particle size increases, and antigen-conjugated solid polymer nanoparticles 20 to $50 \mathrm{~nm}$ in diameter have been shown by multiple studies in mice to more efficiently accumulate in LNs when compared with soluble antigen or with larger nanoparticles (refs. 20-23 and Figure 1). These results correlate well with the estimated approximately 50-nm mean mesh size of connective tissue ECM (24). However, elastic particles (e.g., liposomes) that may deform during transport appear to have different size limits (25). Virus-like particles (VLPs), based on self-assembling proteins (26-28) and synthetic polymer or lipid nanoparticle formulations (21, 29-32), have been demonstrated to enhance vaccine accumulation in LNs and to promote superior cellular and humoral immunity to a variety of antigens in mice relative to soluble forms of protein and peptide vaccines. In addition to size, particle surface chemistry plays a key role in lymphatic trafficking by regulating the tendency toward aggregation in physiological conditions and interactions with cells and/or ECM. For example, liposomes formed of gel-phase cationic lipids containing the danger signal trehalose 6,6-dibehenate, an adjuvant known as CAF01, form a sustained local depot at the injection site that lasts more than 2 weeks, while gel-phase neutral liposomes clear over several days (33). Thus, CAF01 promotes $\mathrm{CD}^{+} \mathrm{T}$ cell responses in humans through attracting and activating APCs at the injection site rather than directly draining to LNs (34). PEGylation of particles is a general strategy to shield surface charge and sterically block aggregation and interactions with the ECM and has often been used to enhance LN accumulation of particulate vaccines $(21,30,35)$.

Targeting APCs in lymphoid organs. Antigens or adjuvants can be designed to target immune cells or endogenous proteins to promote LN accumulation. For example, modification of oligonucleotide adjuvants or peptide antigens with lipid tails designed to bind endogenous albumin was shown to promote substantially enhanced LN uptake (36). By binding to albumin, these low-molecular-weight vaccine components were blocked from disseminating into the blood and instead trafficked efficiently to the lymphatics, leading to accumulation in multiple LN-resident DC subsets. Enhanced LN accumulation translated to greatly increased immunogenicity compared with the equivalent soluble antigen/adjuvant in mice.

DCs are themselves a heterogeneous collection of cells made up of many phenotypic subsets with distinct functions in the adaptive immune response (37). For example, CD11b ${ }^{+}$DCs play an important role in priming $\mathrm{CD} 4^{+} \mathrm{T}$ cells $(38)$, while $\mathrm{CD} 8 \alpha^{+} \mathrm{DCs}$ in mice $\left(\mathrm{CD} 141^{+} \mathrm{XCR} 1^{+} \mathrm{CADM} 1^{+} \mathrm{DCs}\right.$ in humans) are most efficient at presenting antigens acquired from the extracellular environment to $\mathrm{CD}^{+} \mathrm{T}$ cells (37). Targeting specific DC subsets by func- tionalizing vaccines with DC-binding ligands is another approach to enhance LN uptake and vaccine efficacy. Vaccines that target DEC-205 or other cell surface molecules expressed by DCs using antibodies coupled to antigens, nanoparticles, or lentiviral vectors have been shown to enhance LN accumulation and immunogenicity in small animal models $(39,40)$ and to promote both cellular and humoral immunity in humans (41). Thus, antibodies against DEC-205 serve as a proof of concept for the many DC-targeting agents being studied for vaccine delivery $(42,43)$. However, it remains to be determined whether vaccine efficacy can be best achieved by targeting delivery to a precise subset of DCs versus optimizing formulations to activate and antigen-load multiple DC subsets simultaneously.

Separating pharmacokinetics from adjuvant effector properties. Formulation strategies to increase vaccine delivery to LNs can often simultaneously decrease the blood distribution of adjuvants, thereby limiting systemic toxicity. For example, many danger signal compounds, such as $\mathrm{Pam}_{3}$ Cys and monophosphoryl lipid A, are lipids that form micelles or aggregates in water and may interact with proteins and/or reorganize based on the composition of the surrounding solution, making poorly defined products. However, incorporation of these amphiphiles into the bilayers of synthetic liposomes or multilamellar vesicles enables stable adjuvant nanoparticles to be generated with well-defined in vivo biodistribution properties. A prototypical example of this strategy is GlaxoSmithKline's AS01B/E adjuvant formulation, which consists of liposomes incorporating monophosphoryl lipid A and the plant-derived saponin QS-21, a liposomal adjuvant with proven effectiveness in numerous clinical trials, particularly in malaria (44-46). Encapsulation of small-molecule adjuvants in polymer nanoparticles $(47,48)$ or liposomes $(16,49)$ has been shown to eliminate systemic exposure to these compounds, while enhancing accumulation and stimulation of draining LNs in mice and nonhuman primates (NHPs). Direct modifications to small molecules have also been used to alter adjuvant pharmacokinetics: "small-molecule immune potentiator" (SMIP) TLR7 agonists containing aluminum-binding phosphate groups were recently synthesized, which, when mixed with alum, become bound to the adjuvant, focusing their action in the muscle and muscle-draining LNs (50). Alum-binding SMIPs showed much less systemic dissemination/toxicity than their non-alum-binding parent compounds, while exhibiting much greater efficacy for promoting humoral immunity in mice.

\section{Codelivery of antigen and danger signals}

Codelivery and internalization in APCs. In addition to recognition of antigen by the $\mathrm{T}$ cell receptor, $\mathrm{T}$ cells require two additional signals from APCs for maximal activation - costimulation and cytokine stimulation (51). Expression of costimulatory molecules and inflammatory cytokine secretion are triggered in APCs by an encounter with danger signals (52). Studies in mice have suggested that optimal activation of DCs requires direct stimulation by danger signals rather than "in trans" activation by inflammatory cytokines produced by other cells in the tissue $(53,54)$. In addition, optimal humoral responses have been shown in some instances to require PRR signaling in B cells in mice $(48,55)$. During infection, APCs and B cells most often encounter antigen and danger signals 
together as microparticulate (bacteria, fungi) or nanoparticulate (viruses) packages. Notably, optimal antigen processing and presentation following uptake of particulate antigens by DCs in vitro has been shown to require coassociation of appropriate danger signals in the same particle, such that danger signals and antigen are colocalized to the same endosome/phagosome within APCs (ref. 56 and Figure 1). Many examples have been published of murine studies that demonstrate enhanced humoral and cellular immunity elicited by particulate vaccines co-incorporating antigen and danger signal compounds (29, 32, 57-59), and virus-like nanoparticles carrying peptide antigens and CpG DNA have recently been shown to elicit $\mathrm{CD}^{+} \mathrm{T}$ cell responses of magnitudes sufficient for direct ex vivo detection by peptide-MHC tetramer staining in humans (60). A balancing concern, especially for prophylactic vaccines, is the potential of coformulation of antigen and danger signals to trigger autoreactive $\mathrm{T}$ or B cell responses: autoantigen/ danger signal complexes are implicated as drivers of some autoimmune diseases (61). Coencapsulation of antigen and immunosuppressive compounds can be alternatively used to generate particulate vaccines promoting tolerance. For example, polymer nanoparticles containing both antigen and rapamycin were shown to inhibit antigen-specific $\mathrm{T}$ cell activation, increase regulatory $\mathrm{B}$ and $\mathrm{T}$ cells, and inhibit hypersensitivity reactions and autoimmune encephalomyelitis in mouse models (62).

Whether antigen and danger signal coassociation is an absolute requirement for synthetic vaccines has been unclear, as, in some cases, adjuvant compounds delivered in particles mixed with soluble antigen or antigen and adjuvants encapsulated in separate particles have been shown to be effective in driving strong immune responses in mouse and NHP models $(48,49,63)$. However, studies have not yet been carried out to determine whether separately encapsulated danger signals and antigens remain segregated in vivo or, alternatively, if antigen becomes cointernalized with particles or released adjuvant molecules by DCs in situ. Small-molecule adjuvant compounds might disperse rapidly throughout the tissue as they are released from particles at an injection site or in LNs, providing direct stimulation to nearby APCs that internalized antigen without direct co-uptake of distinct particle carriers (64).

A number of strategies have been used to create effective antigen/danger signal co-formulations. Chemical conjugation of adjuvant compounds to protein antigens ensures delivery to the same endocytic compartment, and this approach has been shown to enhance both humoral and cellular immunity in preclinical mouse and NHP models (65-67). Notably, these studies revealed that aggregation of antigen-danger signal conjugates to form particulates was critical to their in vivo efficacy, results which motivated recent studies that demonstrated how polymer-conjugated TLR agonists show both enhanced efficacy and safety when engineered to aggregate into a particulate form (68). The incorporation of antigen or danger signals into particulate vehicles can also be achieved by conjugation of these components to the surface of nanoparticles (69), entrapment within lipid vesicles or capsules (70), or encapsulation within polymer particles (47) or VLPs (60). Materials such as liposomes and poly(lactide-co-glycolide) (PLGA, the polymer used in resorbable sutures) are well-suited as vaccine carriers from a safety perspective due to their long history of safe use in humans.
Targeting multiple danger sensor pathways. All microbes contain multiple danger signals, and the immune response to many live infectious agents has been formally shown to involve multiple PRRs. Perhaps unsurprisingly, certain combinations of danger signals have been shown to trigger strongly synergistic amplification of innate immune activation through PRRs in mouse and human DCs (71). A few studies to date suggest that such synergies may also be relevant for in vivo responses to vaccination. For example, a cancer vaccine based on irradiated flagellin- and ovalbuminexpressing tumor cells was shown to elicit potent $\mathrm{T}$ cell responses in mice, dependent on both TLR5 and the Nod-like receptors NLRC4 and NAIP5 (72). In NHPs, combinations of TLR7/8 and TLR9 agonists enhanced the induction of binding and neutralizing antibody titers against an HIV envelope immunogen (73). High-throughput screening has begun to be applied to the problem of defining optimal danger signal combinations, and in vitro assays predictive of in vivo vaccine performance should enable more facile exploration of the vast parameter space of possible adjuvant combinations (74).

Combination engagement of PRRs can be achieved through the synthesis of chemically linked danger signal ligands (75) or through coformulation strategies. Covalent heterodimers of TLR2 and TLR9 agonists have been shown to elicit enhanced NF-кB expression in DCs relative to stimulation with physical mixtures of these ligands in vitro, and this response may be linked to enforced spatial colocalization of TLRs and their associated adaptor molecules (76). The use of particulate or oil-in-water formulations also facilitates combination delivery of danger signals in mouse and NHP models $(73,77)$. PLGA nanoparticles encapsulating TLR4 and TLR7 agonists were found to synergistically promote strong antibody titers and increase the number of GCs following vaccination in mice, while avoiding the toxicity of the free adjuvant compounds (48). Effective formulation strategies such as this will be key to safely implementing synergistic danger signal compounds as adjuvants. A key question for the future is whether the synergies observed between TLR agonists and other danger signals in the studies to date will still be manifest and important when danger signals are optimally formulated for LN delivery and APC uptake.

\section{Optimizing antigen display to B cells}

The majority of licensed vaccines are thought to protect through the generation of neutralizing antibodies (78), and thus strategies to promote the humoral response are of key importance in vaccine development. Because multivalent $\mathrm{B}$ cell receptor (BCR) engagement and clustering promotes B cell activation (79), vaccine formulations can play an important role in regulating the presentation of antigens to B cells in a manner that promotes BCR aggregation. Molecules that can elicit B cell activation and antibody production in the absence of $\mathrm{T}$ cell help (e.g., bacterial polysaccharides) are known as T-independent antigens, while most peptide and protein antigens are $\mathrm{T}$-dependent and require both $\mathrm{BCR}$ triggering and interactions of B cells with $\mathrm{CD} 4^{+}$Th cells. Early studies using small-molecule antigens termed haptens demonstrated that these $\mathrm{T}$-independent epitopes were only immunogenic in mice when presented as multimers of at least 10 to 20 haptens linked to a synthetic polymer backbone (80). Polymers functionalized with haptens show an increase in BCR clustering and calcium flux in 
vitro, suggesting that B cells differentiate antigens based on the epitope density (81). Similarly, polysaccharide antigens arrayed on polymer nanoparticles have been shown to elicit enhanced humoral responses compared with soluble forms of the same antigens in mice (82). Generally, these results suggest that highdensity antigens arrayed on functionalized polymers or particles yield improved $\mathrm{T}$-independent antibody responses.

For T-dependent protein/peptide antigens, increasing valency (through multimerization, coupling to polymers/carrier proteins, or display from nanoparticles) has been shown to lead to increasing B cell triggering, antigen internalization, and presentation to Th cells both in vitro and in vivo in mice (83-85). Dimeric and trimeric forms of a model protein antigen bound to B cells and were presented on MHC II complexes, while monomeric versions did not lead to epitope presentation in vitro (83). Antigen organization - whether epitopes are displayed in a dense or sparse array and whether they are held in a rigid or flexible orientation - also affects B cell responses in mice (ref. 86 and Figure 1). For example, VLPs displaying peptide epitopes were shown to only induce IgG responses in vivo when the antigen was displayed at high density (84). This response can be further amplified if the VLPs incorporate TLR agonists in a manner dependent on TLR signaling in $B$ cells and high-density display of antigen (59). These results may reflect the importance of antigen spacing, the total number of antigens per particle, or both but are consistent with the idea that $\mathrm{B}$ cells have evolved to optimally recognize viruses and bacteria that often express dense, rigidly arrayed repetitive copies of binding and entry proteins at their surfaces. High-density display of epitopes by VLPs has been shown to in fact be capable of overcoming B cell tolerance to neo-self antigens in mice (87). The licensed human papilloma virus vaccines, hepatitis $\mathrm{B}$ vaccine, and the most-studied malaria vaccine candidate, RTS,S, are all examples of vaccines based on the use of self-assembled VLPs that exploit these principles (88). Recently, it has been demonstrated that multivalent antigens formed by supramolecular assembly of peptides from ovalbumin or model protein antigens into nanoscale-diameter fibers (presenting thousands of copies of antigen per fiber) elicit robust humoral immune responses in mice $(89,90)$. Interestingly, nanofibers carrying a malaria antigen elicited robust T-dependent antibody responses in a manner dependent on MyD88 signaling but independent of the NALP3 inflammasome (91). Vaccines displaying multivalent antigens at the surfaces of synthetic nanoparticles (35), peptide micelles (92), or engineered protein/peptide particles $(26,93)$ or on soluble polymer backbones $(94)$ have shown promise in preclinical studies in mice. Notably, many of these strategies for optimizing antigen display to B cells are also expected to enhance overall antigen accumulation in LNs (Figure 1).

The presentation of antigens to B cells in vivo depends on the route taken by the antigen to accumulate in the LN. Unlike DCs that can interact with and become activated by the vaccine at the injection site, B cell stimulation is confined to the LNs during the primary response. B cells often encounter particulate antigens at the border between follicles and the subcapsular sinus of LNs through macrophages that initially acquire and then "hand off" the antigens (95-97). In contrast, influenza particles are captured in the medulla of LNs (98). For small soluble antigens, B cells appear to interact directly with the antigen in the follicular region
(99). Using intravital microscopy, smaller proteins (14 kDa) were observed to diffuse from the subcapsular sinus into the B cell follicular region, while larger proteins $(450 \mathrm{kDa})$ were taken up by subcapsular sinus macrophages and then presented to follicular B cells (100). These processes are alternatives to DCs capturing and trafficking antigen to the LNs from the periphery (101). An important unanswered question is how these many different pathways for antigen delivery to B cells influence subsequent B cell triggering and interactions with Th cells.

\section{Programming vaccine kinetics}

Natural infections versus artificial immunizations. Chronic infections induce sustained high levels of antigen and inflammatory cues, such as IL-1, IL-6, TNF- $\alpha$, caspase-1, and type I IFNs, for many months, leading to major defects in the immune response (102). By contrast, during "acute" viral infections, the majority of infectious virus is often cleared by 7 days, but residual antigen/ viral nucleic acids can often be detected for 4 to 8 weeks, with clear effects on the immune response (103-105). Persistent antigen/inflammation exposure in draining LNs over periods of at least several days can be important for maximizing the immune response, enhancing the differentiation of follicular Th cells (106, 107), enhancing GC induction (35), and producing an optimal cytokine milieu (108). The duration and magnitude of antigen and adjuvant exposure during priming of naive lymphocytes or boosting of memory cells is known to play a significant role in determining the degree of clonal expansion (109-111), fate decisions between different functional phenotypes (Th subsets or plasma cells versus memory cell differentiation of B cells; refs. 106, 112), and the quality of memory established $(113,114)$. Further, studies examining the role of temporal dosing profiles through repeated daily injections of antigen/adjuvant showed that exponentially increasing doses of an antigen and adjuvant over the course of a week could greatly increase $\mathrm{T}$ cell responses to a model peptide vaccine in mice (108).

These data suggest that strategies to control the kinetics of vaccine exposure could have a substantial beneficial effect on the response to immunization. However, unformulated soluble antigens/adjuvants rapidly flush through draining LNs within hours and are quickly cleared by lymph flow $(35,99)$. There is a general belief that classic adjuvants, such as alum and oil-in-water emulsions, act to create depots of antigen at the injection site, suggesting that existing vaccines may already achieve some level of sustained vaccine exposure. However, the oil-in-water emulsion MF59 has been shown not to alter antigen clearance from injection sites or persistence in draining LNs (11). Alum appears to form a depot with some antigens (12) but not others (115) and likely undergoes desorption of some antigens under in vivo conditions (116). Recent studies have further demonstrated that, following alum immunization, the injection site can be surgically removed 2 hours after injection with no effect on the humoral immune response, suggesting that depot formation is not a critical part of alum's mechanism of action $(117,118)$.

Engineering vaccine kinetics. In one of the earliest studies of the use of synthetic polymer matrices to obtain sustained release of biologics, Robert Langer's group demonstrated that nonbiodegradable poly(ethylene-co-vinyl acetate) implants could release 
A

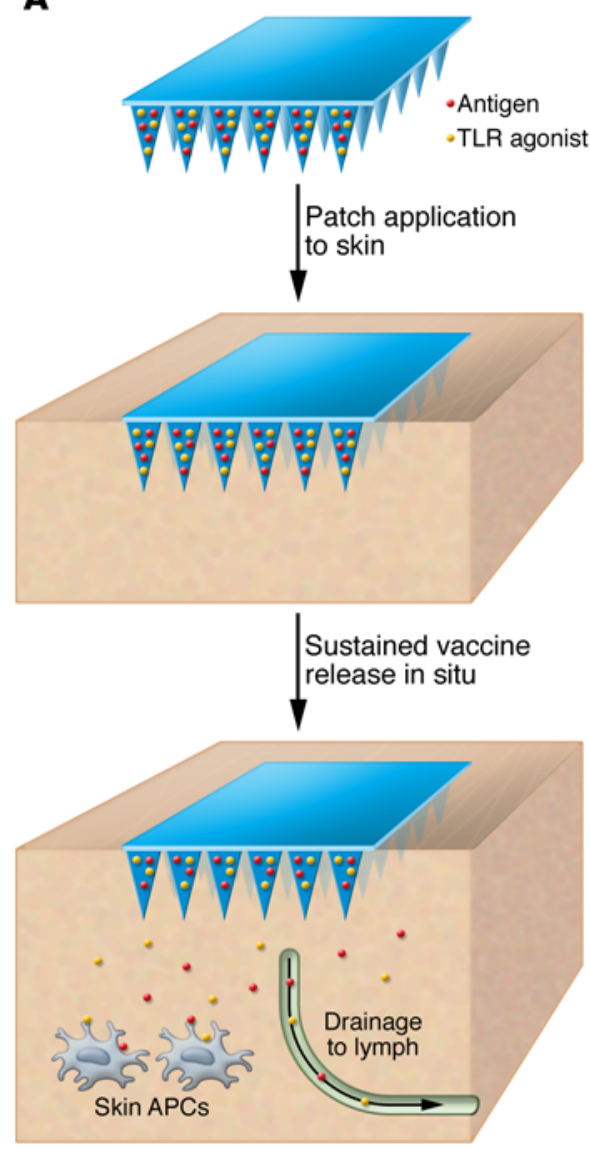

B

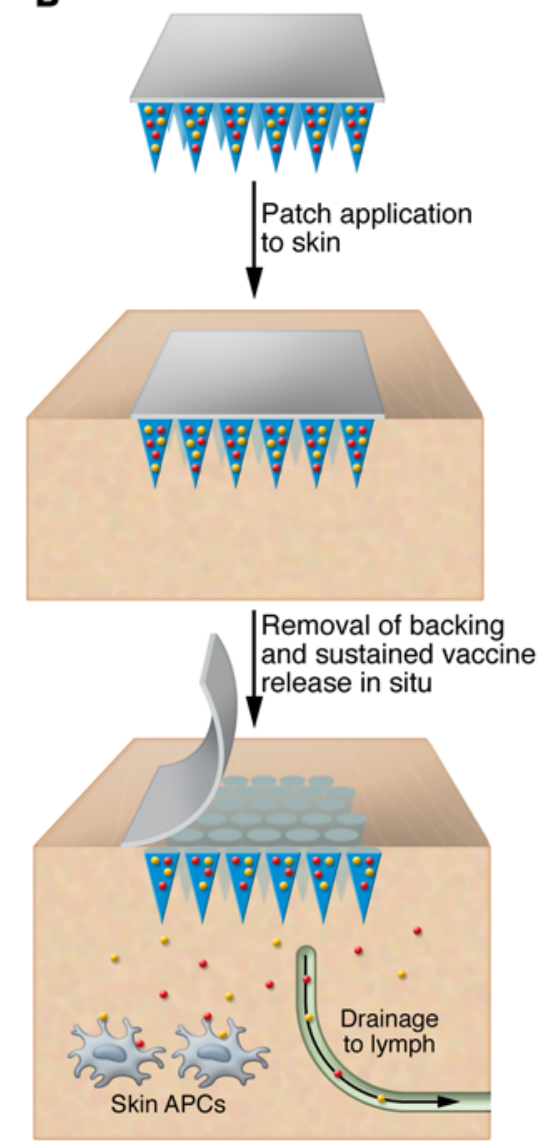

C

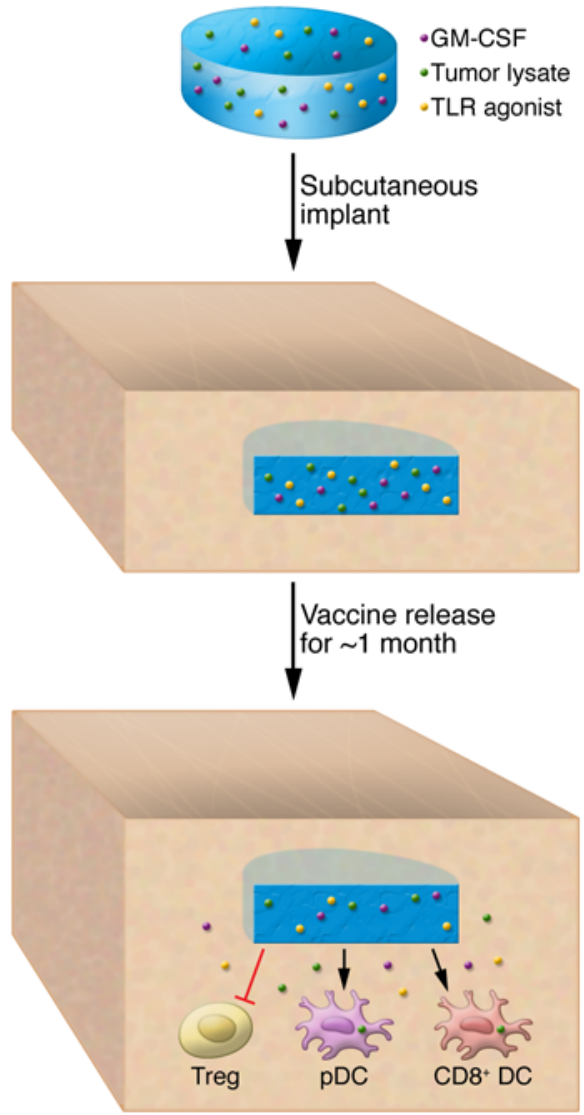

Figure 2. Programming vaccine kinetics. (A) Microneedle arrays composed of a swellable or slowly dissolving polymer that releases entrapped vaccine over time. (B) Implantable microneedles detach from a dissolving backing following brief application to skin and then slowly release vaccine in situ. (C) Porous PLGA sponges that are loaded with tumor lysates, the cytokine granulocyte-macrophage CSF (CM-CSF), and TLR agonists are implanted subcutaneously. DC precursors are attracted to the scaffold, differentiated, activated, and loaded with antigen in situ as controlled release from the implant takes place for up to 1 month. Immunoregulatory pathways that involve Tregs are also inhibited. pDC, plasmacytoid DC.

protein antigens over up to a month in vivo in mice, leading to antibody responses equivalent to multiple injections with Freund's adjuvant (119). To achieve more practical formulations for prophylactic vaccines, biodegradable PLGA microspheres used to encapsulate and slowly release vaccine antigens were pursued by many groups in the 1990s; however, chronic issues of antigen degradation within the acidic microclimate of these polymer particles, difficulties in manufacturing scale-up, and challenges of demonstrating superiority of these formulations over alum have contributed to the fact that no biodegradable polymer formulation of encapsulated vaccine antigens has yet to move forward into advanced clinical trials (120). However, PLGA particle formulations of danger signals or tolerogenic compounds may be an effective strategy to influence adjuvant kinetics in vaccination, and such approaches are under development in preclinical models $(48,62,121)$.

Several additional new technologies offer the potential to control vaccine kinetics in prophylactic or therapeutic vaccines. Microneedle skin patches, comprising an array of solid pyramidal or cylindrical projections that are each a fraction of a millimeter in height and several hundred microns at their base, are designed to mechanically perforate the stratum corneum to enter the viable epidermis and/or upper dermis upon application to the skin (Fig- ure 2, A and B). Microneedles can be coated with dried vaccine formulations, or the microneedles themselves can comprise dissolving polymers that release the vaccine upon application to the skin. Vaccine administration via microneedles provides several key advantages over traditional intramuscular injections by targeting the abundant repertoire of immune cells in the skin, allowing for minimal pain and discomfort, and providing a way to encapsulate the bioactive molecules in a stable, lyophilized state prior to use (122). Several types of microneedle patches that can tailor vaccine kinetics have been designed and tested in mice, NHPs, and early clinical trials, including microneedles composed of polymers that swell or dissolve at controlled rates when applied to the skin, releasing encapsulated vaccines (refs. 123-125 and Figure $2 \mathrm{~A})$; microneedles composed of polymer microparticles or nanoparticles carrying encapsulated vaccine supported in a dissolving matrix, which rapidly deposit vaccine-loaded particles into the skin for subsequent tunable release kinetics from the implanted particles $(126,127)$; and microneedles composed of a solid tip or polymer coating on a dissolving base, in which the vaccine-releasing tip is rapidly implanted into the skin upon patch application (refs. 126, 128, 129, and Figure 2B). A particularly interesting example is the encapsulation of vaccine antigens within silk protein-based 
microneedles, as silk protein has been shown to thermostabilize vaccines in a dried state (130), and silk matrices can be designed to release vaccines with extended kinetics in the skin, promoting $\mathrm{T}$ cell and humoral responses following vaccination (128).

A second approach that is promising, particularly for the development of therapeutic vaccines, is the formulation of antigens and danger signals together within porous PLGA polymer sponges, which are implanted into the skin. These resorbable vaccine scaffolds orchestrate the attraction, activation, and antigen loading of APCs through tunable kinetics of antigen and adjuvant release at the implant site in mice (refs. 131, 132, and Figure 2C). In preclinical models of therapeutic cancer vaccination, these structures elicited potent antitumor $\mathrm{T}$ cell responses that were superior to a leading clinical cell-based cancer vaccine. These responses were mediated by optimal programming of responding DCs (133). This PLGA scaffold-based vaccine approach has recently entered phase I clinical testing in patients with melanoma.

\section{Conclusions}

The first vaccines based on live, attenuated microbes were developed empirically with a poor understanding of how they or their disease-causing source pathogens interacted with the immune system. We now understand that pathogens such as viruses are often of a size that is optimal for efficient dissemination through lymphatics for collection in LNs; that microbes contain many components that are recognized by innate immune PRRs, which direct the immune response; that B cells and APCs are highly attuned to the particulate structure and repetitive display of surface proteins/sugars on microbes; and that the duration of antigen and inflammatory cues encountered by the immune system during infection strongly shape the resulting immune response. Controllably mimicking these features of live infections in a tailored manner to induce immunity safely and efficiently with synthetic vaccines is a grand challenge being tackled by current efforts in vaccine development. To this end, vaccine formulations comprising engineered materials that control antigen and adjuvant biodistribution, regulate uptake of vaccine by APCs, optimize triggering of antigen-specific B cells, and influence vaccine kinetics will all have a role to play in the design of future vaccines. These advances may be critical to the development of vaccines that overcome the significant challenges presented by diseases such as HIV, tuberculosis, and cancer or that can be used to treat autoimmunity or promote therapeutic tolerance.

\section{Acknowledgments}

We apologize to colleagues whose relevant work may not have been cited due to space constraints in this brief Review. This work was supported in part by the NIH (awards UM1AI100663, AI104715, AI091693, and AI095109); the Ragon Institute of MGH, MIT and Harvard; the Bill and Melinda Gates Foundation; the V Foundation; the Bridge Project of the Koch Institute; the Dana-Farber/Harvard Cancer Center; and the US Army Research Laboratory and the US Army Research Office through the Institute for Soldier Nanotechnologies (contract W911NF-13-D-0001). A.C. Zmolek is supported in part by the National Science Foundation Graduate Research Fellowship (grant 1122374). The content is solely the responsibility of the authors and does not necessarily represent the official views of the NIH. D.J. Irvine is an investigator of the Howard Hughes Medical Institute.

Address correspondence to: Darrell J. Irvine, David H. Koch Institute for Integrative Cancer Research, Massachusetts Institute of Technology, 76-261, 500 Main Street, Cambridge, Massachusetts 02142, USA. Phone: 617.452.4174; E-mail: djirvine@mit.edu.
1. Koff WC, et al. Accelerating next-generation vaccine development for global disease prevention. Science. 2013;340(6136):1232910.

2. Walker BD, Burton DR. Toward an AIDS vaccine. Science. 2008;320(5877):760-764.

3. Nabel GJ. Designing tomorrow's vaccines. N Engl JMed. 2013;368(6):551-560.

4. Di Pasquale A, Preiss S, Da Silva FT, Garcon N. Vaccine adjuvants: from 1920 to 2015 and beyond. Vaccines. 2015;3(2):320-343.

5. Reed SG, Orr MT, Fox CB. Key roles of adjuvants in modern vaccines. Nat Med. 2013;19(12):1597-1608.

6. van Duin D, Medzhitov R, Shaw AC. Triggering TLR signaling in vaccination. Trends Immunol. 2006;27(1):49-55.

7. Bojang KA, et al. Efficacy of RTS,S/ASO2 malaria vaccine against Plasmodium falciparum infection in semi-immune adult men in The Gambia: a randomised trial. Lancet. 2001;358(9297):1927-1934.

8. Lewis GK, DeVico AL, Gallo RC. Antibody persistence and T-cell balance: two key factors confronting HIV vaccine development. Proc Natl Acad Sci U S A. 2014;111(44):15614-15621.

9. Burton DR, Poignard P, Stanfield RL, Wilson IA. Broadly neutralizing antibodies present new prospects to counter highly antigenically diverse viruses. Science. 2012;337(6091):183-186.

10. O'Hagan DT, Ott GS, De Gregorio E, Seubert A.
The mechanism of action of MF59 - an innately attractive adjuvant formulation. Vaccine. 2012;30(29):4341-4348.

11. Dupuis M, McDonald DM, Ott G. Distribution of adjuvant MF59 and antigen gD2 after intramuscular injection in mice. Vaccine. 1999; 18(5-6):434-439.

12. De Veer M, Kemp J, Chatelier J, Elhay MJ, Meeusen EN. The kinetics of soluble and particulate antigen trafficking in the afferent lymph, and its modulation by aluminum-based adjuvant. Vaccine. 2010;28(40):6597-6602.

13. Cantisani R, et al. Vaccine adjuvant MF59 promotes retention of unprocessed antigen in lymph node macrophage compartments and follicular dendritic cells. JImmunol. 2015;194(4):1717-1725.

14. Supersaxo A, Hein WR, Steffen H. Effect of molecular weight on the lymphatic absorption of water-soluble compounds following subcutaneous administration. Pharm Res. 1990;7(2):167-169.

15. Miller NE, et al. Secretion of adipokines by human adipose tissue in vivo: partitioning between capillary and lymphatic transport. Am J Physiol Endocrinol Metab. 2011;301(4):E659-E667.

16. Smirnov D, Schmidt JJ, Capecchi JT, Wightman PD. Vaccine adjuvant activity of 3M-052: an imidazoquinoline designed for local activity without systemic cytokine induction. Vaccine.
2011;29(33):5434-5442.

17. Kourtis IC, et al. Peripherally administered nanoparticles target monocytic myeloid cells, secondary lymphoid organs and tumors in mice. PLOS One. 2013;8(4):e61646.

18. Hubbell JA, Thomas SN, Swartz MA. Materials engineering for immunomodulation. Nature. 2009;462(7272):449-460.

19. Bachmann MF, Jennings GT. Vaccine delivery: a matter of size, geometry, kinetics and molecular patterns. Nat Rev Immunol. 2010;10(11):787-796.

20. Fifis T, et al. Size-dependent immunogenicity: therapeutic and protective properties of nano-vaccines against tumors. JImmunol. 2004;173(5):3148.

21. Reddy ST, et al. Exploiting lymphatic transport and complement activation in nanoparticle vaccines. Nat Biotechnol. 2007;25(10):1159-1164.

22. Reddy ST, Rehor A, Schmoekel HG, Hubbell JA, Swartz MA. In vivo targeting of dendritic cells in lymph nodes with poly(propylene sulfide) nanoparticles. JControl Release. 2006;112(1):26-34.

23. Manolova V, Flace A, Bauer M, Schwarz K, Saudan P, Bachmann MF. Nanoparticles target distinct dendritic cell populations according to their size. Eur J Immunol. 2008;38(5):1404-1413.

24. Griffith LG, Swartz MA. Capturing complex 3D tissue physiology in vitro. Nat Rev Mol Cell Biol. 
2006;7(3):211-224.

25. Oussoren C, Zuidema J, Crommelin D, Storm G. Lymphatic uptake and biodistribution of liposomes after subcutaneous injection. Biochim Biophys Acta. 1997;1328(2):261-272.

26. Kanekiyo M, et al. Self-assembling influenza nanoparticle vaccines elicit broadly neutralizing H1N1 antibodies. Nature. 2013;499(7456):102-106.

27. Link A, Zabel F, Schnetzler Y, Titz A, Brombacher F, Bachmann MF. Innate immunity mediates follicular transport of particulate but not soluble protein antigen. J Immunol. 2012;188(8):3724-3733.

28. Chackerian B, Lowy DR, Schiller JT. Conjugation of a self-antigen to papillomavirus-like particles allows for efficient induction of protective autoantibodies. JClin Invest. 2001;108(3):415

29. Moon JJ, et al. Interbilayer-crosslinked multilamellar vesicles as synthetic vaccines for potent humoral and cellular immune responses. Nat Mater. 2011;10(3):243-251.

30. Nembrini C, et al. Nanoparticle conjugation of antigen enhances cytotoxic T-cell responses in pulmonary vaccination. Proc Natl Acad Sci U S A. 2011;108(44):E989-E997.

31. Li AV, et al. Generation of effector memory T cell-based mucosal and systemic immunity with pulmonary nanoparticle vaccination. Sci Transl Med. 2013;5(204):204ra130.

32. Demento SL, et al. TLR9-targeted biodegradable nanoparticles as immunization vectors protect against West Nile encephalitis. JImmunol. 2010;185(5):2989-2997.

33. Henriksen-Lacey M, et al. Liposomal cationic charge and antigen adsorption are important properties for the efficient deposition of antigen at the injection site and ability of the vaccine to induce a CMI response. JControl Release. 2010;145(2):102-108.

34. van Dissel JT, et al. A novel liposomal adjuvant system, CAF01, promotes long-lived Mycobacterium tuberculosis-specific T-cell responses in human. Vaccine. 2014;32(52):7098-7107.

35. Moon JJ, Suh H, Li AV, Ockenhouse CF, Yadava A, Irvine DJ. Enhancing humoral responses to a malaria antigen with nanoparticle vaccines that expand Tfh cells and promote germinal center induction. Proc Natl Acad Sci U S A. 2012;109(4):1080-1085.

36. Liu $\mathrm{H}$, et al. Structure-based programming of lymph-node targeting in molecular vaccines. Nature. 2014;507(7493):519-522.

37. Mildner A, Jung S. Development and function of dendritic cell subsets. Immunity. 2014;40(5):642-656.

38. Dudziak D, et al. Differential antigen processing by dendritic cell subsets in vivo. Science. 2007;315(5808):107-111.

39. Tacken PJ, et al. Targeted delivery of TLR ligands to human and mouse dendritic cells strongly enhances adjuvanticity. Blood. 2011;118(26):6836-6844.

40. Yang L, et al. Engineered lentivector targeting of dendritic cells for in vivo immunization. Nat Biotechnol. 2008;26(3):326-334.

41. Dhodapkar MV, et al. Induction of antigen-specific immunity with a vaccine targeting NY-ESO-1 to the dendritic cell receptor DEC-
205. Sci Transl Med. 2014;6(232):232ra251.

42. Caminschi I, Shortman K. Boosting antibody responses by targeting antigens to dendritic cells. Trends Immunol. 2012;33(2):71-77.

43. Tacken PJ, de Vries IJM, Torensma R, Figdor CG. Dendritic-cell immunotherapy: from ex vivo loading to in vivo targeting. Nat Rev Immunol. 2007;7(10):790-802.

44. Garçon N, Chomez P, Van Mechelen M. GlaxoSmithKline Adjuvant Systems in vaccines: concepts, achievements and perspectives. Expert Rev Vaccines. 2007;6(5):723-739.

45. Bejon P, et al. Efficacy of RTS, S/ASO1E vaccine against malaria in children 5 to 17 months of age. N Engl JMed. 2008;359(24):2521-2532.

46. Olotu A, et al. Efficacy of RTS,S/ASO1E malaria vaccine and exploratory analysis on anti-circumsporozoite antibody titres and protection in children aged 5-17 months in Kenya and Tanzania: a randomised controlled trial. Lancet Infect Dis. 2011;11(2):102-109.

47. Ilyinskii PO, et al. Adjuvant-carrying synthetic vaccine particles augment the immune response to encapsulated antigen and exhibit strong local immune activation without inducing systemic cytokine release. Vaccine. 2014;32(24):2882-2895.

48. Kasturi SP, et al. Programming the magnitude and persistence of antibody responses with innate immunity. Nature. 2011;470(7335):543-547.

49. Hanson MC, et al. Nanoparticulate STING agonists are potent lymph node-targeted vaccine adjuvants. JClin Invest. 2015;125(6):2352-2546.

50. Wu TY-H, et al. Rational design of small molecules as vaccine adjuvants. Sci Transl Med. 2014;6(263):263ra160.

51. Curtsinger JM, Mescher MF. Inflammatory cytokines as a third signal for T cell activation. Curr Opin Immunol. 2010;22(3):333-340.

52. Pulendran B, Ahmed R. Immunological mechanisms of vaccination. Nat Immunol. 2011;12(6):509-517.

53. Desch AN, et al. Dendritic cell subsets require cis-activation for cytotoxic CD8 T-cell induction. Nat Commun. 2014;5:4674.

54. Sporri R, Reis e Sousa C. Inflammatory mediators are insufficient for full dendritic cell activation and promote expansion of $\mathrm{CD} 4^{+} \mathrm{T}$ cell populations lacking helper function. Nat Immunol. 2005;6(2):163-170.

55. Pasare C, Medzhitov R. Control of B-cell responses by Toll-like receptors. Nature. 2005;438(7066):364-368.

56. Blander JM, Medzhitov R. Toll-dependent selection of microbial antigens for presentation by dendritic cells. Nature. 2006;440(7085):808-812.

57. Steers NJ, Peachman KK, McClain S, Alving CR, Rao M. Liposome-encapsulated HIV-1 Gag p24 containing lipid A induces effector $\mathrm{CD} 4^{+} \mathrm{T}$-cells, memory $\mathrm{CD} 8^{+} \mathrm{T}$-cells, and pro-inflammatory cytokines. Vaccine. 2009;27(49):6939-6949.

58. Stano A, van der Vlies AJ, Martino MM, Swartz MA, Hubbell JA, Simeoni E. PPS nanoparticles as versatile delivery system to induce systemic and broad mucosal immunity after intranasal administration. Vaccine. 2011;29(4):804-812.

59. Hou B, et al. Selective utilization of Toll-like receptor and MyD88 signaling in B cells for enhancement of the antiviral germinal center response. Immunity. 2011;34(3):375-384.

60. Goldinger SM, et al. Nano-particle vaccination combined with TLR-7 and -9 ligands triggers memory and effector CD8(+) T-cell responses in melanoma patients. Eur J Immunol. 2012;42(11):3049-3061.

61. Marshak-Rothstein A, Rifkin IR. Immunologically active autoantigens: the role of toll-like receptors in the development of chronic inflammatory disease. Annu Rev Immunol. 2007;25:419-441.

62. Maldonado RA, et al. Polymeric synthetic nanoparticles for the induction of antigen-specific immunological tolerance. Proc Natl Acad Sci US A. 2015;112(2):E156-E165.

63. Malyala P, et al. The potency of the adjuvant, CpG oligos, is enhanced by encapsulation in PLG microparticles. JPharm Sci. 2008;97(3):1155-1164.

64. Jewell CM, Lopez SC, Irvine DJ. In situ engineering of the lymph node microenvironment via intranodal injection of adjuvant-releasing polymer particles. Proc Natl Acad Sci U S A. 2011;108(38):15745-15750.

65. Wille-Reece U, et al. HIV Gag protein conjugated to a Toll-like receptor $7 / 8$ agonist improves the magnitude and quality of $\mathrm{Th} 1$ and $\mathrm{CD} 8^{+} \mathrm{T}$ cell responses in nonhuman primates. Proc Natl Acad Sci U S A. 2005;102(42):15190-15194.

66. Kastenmüller K, et al. Protective T cell immunity in mice following protein-TLR7/8 agonist-conjugate immunization requires aggregation, type I IFN, and multiple DC subsets. JClin Invest. 2011;121(5):1782.

67. Oh JZ, Kurche JS, Burchill MA, Kedl RM. TLR7 enables cross-presentation by multiple dendritic cell subsets through a type I IFN-dependent pathway. Blood.2011;118(11):3028-3038.

68. Lynn GM, et al. In vivo characterization of the physicochemical properties of polymer-linked TLR agonists that enhance vaccine immunogenicity. Nat Biotechnol. 2015;33(11):1201-1210.

69. de Titta A, et al. Nanoparticle conjugation of $\mathrm{CpG}$ enhances adjuvancy for cellular immunity and memory recall at low dose. Proc Natl Acad Sci US A. 2013;110(49):19902-19907.

70. Moon JJ, et al. Interbilayer-crosslinked multilamellar vesicles as synthetic vaccines for potent humoral and cellular immune responses. Nat Mater. 2011;10(3):243-251.

71. Napolitani G, Rinaldi A, Bertoni F, Sallusto F, Lanzavecchia A. Selected Toll-like receptor agonist combinations synergistically trigger a Thelper type 1-polarizing program in dendritic cells. Nat Immunol. 2005;6(8):769-776.

72. Garaude J, Kent A, van Rooijen N, Blander JM. Simultaneous targeting of toll-and nod-like receptors induces effective tumorspecific immune responses. Sci Transl Med. 2012;4(120):120ra116.

73. Moody MA, et al. Toll-like receptor 7/8 (TLR7/8) and TLR9 agonists cooperate to enhance HIV-1 envelope antibody responses in rhesus macaques. JVirol. 2014;88(6):3329-3339.

74. Garcia-Cordero JL, Nembrini C, Stano A, Hubbell JA, Maerkl SJ. A high-throughput nanoimmunoassay chip applied to large-scale vaccine adjuvant screening. Integr Biol (Camb). 2013;5(4):650-658. 
75. Shukla NM, Mutz CA, Malladi SS, Warshakoon HJ, Balakrishna R, David SA. Toll-like receptor (TLR)-7 and-8 modulatory activities of dimeric imidazoquinolines. J Med Chem. 2012;55(3):1106-1116.

76. Mancini RJ, Tom JK, Esser-Kahn AP. Covalently coupled immunostimulant heterodimers. Angew Chem Int Ed Engl. 2014;126(1):193-196.

77. Orr MT, et al. A dual TLR agonist adjuvant enhances the immunogenicity and protective efficacy of the tuberculosis vaccine antigen ID93. PLoS One. 2014;9(1):e83884.

78. Amanna IJ, Slifka MK. Contributions of humoral and cellular immunity to vaccine-induced protection in humans. Virology. 2011;411(2):206-215.

79. Mattila PK, et al. The actin and tetraspanin networks organize receptor nanoclusters to regulate B cell receptor-mediated signaling. Immunity. 2013;38(3):461-474.

80. Dintzis H, Dintzis R, Vogelstein B. Molecular determinants of immunogenicity: the immunon model of immune response. Proc Natl Acad Sci U S A. 1976;73(10):3671-3675.

81. Puffer EB, Pontrello JK, Hollenbeck JJ, Kink JA, Kiessling LL. Activating B cell signaling with defined multivalent ligands. ACS Chem Biol. 2007;2(4):252-262.

82. Anish C, Khan N, Upadhyay AK, Sehgal D, Panda AK. Delivery of polysaccharides using polymer particles: implications on size-dependent immunogenicity, opsonophagocytosis, and protective immunity. Mol Pharm. 2014;11(3):922-937.

83. Kim Y-M, Pan JY-J, Korbel GA, Peperzak V, Boes $\mathrm{M}$, Ploegh HL. Monovalent ligation of the B cell receptor induces receptor activation but fails to promote antigen presentation. Proc Natl Acad Sci US A. 2006;103(9):3327-3332.

84. Jegerlehner A, Storni T, Lipowsky G, Schmid M, Pumpens P, Bachmann MF. Regulation of $\operatorname{IgG}$ antibody responses by epitope density and CD21-mediated costimulation. Eur J Immunol. 2002;32(11):3305-3314

85. Temchura VV, Kozlova D, Sokolova V, Uberla K, Epple M. Targeting and activation of antigenspecific B-cells by calcium phosphate nanoparticles loaded with protein antigen. Biomaterials. 2014;35(23):6098-6105.

86. Bachmann MF, Rohrer UH, Kundig TM, Burki K, Hengartner $\mathrm{H}$, Zinkernagel RM. The influence of antigen organization on B cell responsiveness. Science. 1993;262(5138):1448-1451.

87. Chackerian B, Durfee MR, Schiller JT. Viruslike display of a neo-self antigen reverses $B$ cell anergy in a B cell receptor transgenic mouse model. JImmunol. 2008;180(9):5816-5825.

88. Ross TM, et al. Virus-like particle vaccines: advantages and challenges. In: Singh M, Srivastava IK, eds. Development of Vaccines: From Discovery to Clinical Testing. Hoboken, New Jersey, USA: John Wiley \& Sons, Inc; 2011:95-124.

89. Rudra JS, Tian YF, Jung JP, Collier JH. A self-assembling peptide acting as an immune adjuvant. Proc Natl Acad Sci U S A. 2010;107(2):622-627.

90. Hudalla GA, et al. A self-adjuvanting supramolecular vaccine carrying a folded protein antigen. Adv Healthc Mater. 2013;2(8):1114-1119.

91. Rudra JS, et al. Self-assembled peptide nanofibers raising durable antibody responses against a malaria epitope. Biomaterials. 2012;33(27):6476-6484.

92. Boato F, Thomas RM, Ghasparian A, FreundRenard A, Moehle K, Robinson JA. Synthetic virus-like particles from self-assembling coiledcoil lipopeptides and their use in antigen display to the immune system. Angew Chem Int Ed Engl. 2007;119(47):9173-9176.

93. Jardine JG, et al. HIV-1 vaccines. Science. 2015;349(6244):156-161.

94. Zhang F, Lu YJ, Malley R. Multiple antigenpresenting system (MAPS) to induce comprehensive B- and T-cell immunity. Proc Natl Acad Sci U S A. 2013;110(33):13564-13569.

95. Carrasco YR, Batista FD. B cells acquire particulate antigen in a macrophage-rich area at the boundary between the follicle and the subcapsular sinus of the lymph node. Immunity. 2007;27(1):160-171.

96. Junt $\mathrm{T}$, et al. Subcapsular sinus macrophages in lymph nodes clear lymph-borne viruses and present them to antiviral B cells. Nature. 2007;450(7166):110-114.

97. Phan TG, Grigorova I, Okada T, Cyster JG. Subcapsular encounter and complement-dependent transport of immune complexes by lymph node $\mathrm{B}$ cells. Nat Immunol. 2007;8(9):992-1000.

98. Woodruff MC, et al. Trans-nodal migration of resident dendritic cells into medullary interfollicular regions initiates immunity to influenza vaccine. J Exp Med. 2014;211(8):1611-1621.

99. Pape KA, Catron DM, Itano AA, Jenkins MK. The humoral immune response is initiated in lymph nodes by B cells that acquire soluble antigen directly in the follicles. Immunity. 2007;26(4):491-502.

100. Roozendaal R, et al. Conduits mediate transport of low-molecular-weight antigen to lymph node follicles. Immunity. 2009;30(2):264-276.

101. Kissenpfennig A, et al. Dynamics and function of langerhans cells in vivo: dermal dendritic cells colonize lymph node areasdistinct from slower migrating langerhans cells. Immunity. 2005;22(5):643-654.

102. Wherry EJ, et al. Molecular signature of $\mathrm{CD} 8^{+} \mathrm{T}$ cell exhaustion during chronic viral infection. Immunity. 2007;27(4):670-684.

103. Lin WH, Kouyos RD, Adams RJ, Grenfell BT, Griffin DE. Prolonged persistence of measles virus RNA is characteristic of primary infection dynamics. Proc Natl Acad Sci U S A. 2012;109(37):14989-14994.

104.Kim TS, Hufford MM, Sun J, Fu YX, Braciale TJ. Antigen persistence and the control of local $\mathrm{T}$ cell memory by migrant respiratory dendritic cells after acute virus infection. JExp Med. 2010;207(6):1161-1172.

105. Turner DL, Cauley LS, Khanna KM, Lefrancois L. Persistent antigen presentation after acute vesicular stomatitis virus infection. J Virol. 2007;81(4):2039-2046.

106.Baumjohann D, et al. Persistent antigen and germinal center $\mathrm{B}$ cells sustain $\mathrm{T}$ follicular helper cell responses and phenotype. Immunity. 2013;38(3):596-605.

107. Benson RA, MacLeod MK, Hale BG, Patakas A, Garside P, Brewer JM. Antigen presentation kinetics control $\mathrm{T}$ cell/dendritic cell interactions and follicular helper $\mathrm{T}$ cell generation in vivo. Elife. 2015;4:e06994.

108. Johansen $P$, et al. Antigen kinetics determines immune reactivity. Proc Natl Acad Sci US A. 2008;105(13):5189-5194.

109. Joshi NS, et al. Inflammation directs memory precursor and short-lived effector $\mathrm{CD} 8^{+} \mathrm{T}$ cell fates via the graded expression of T-bet transcription factor. Immunity. 2007;27(2):281-295.

110.Zinkernagel RM. Localization dose and time of antigens determine immune reactivity. Semin Immunol. 2000;12(3):163-171.

111. Blair DA, et al. Duration of antigen availability influences the expansion and memory differentiation of T cells. JImmunol. 2011;187(5):2310-2321.

112. Paus D, Phan TG, Chan TD, Gardam S, Basten A, Brink R. Antigen recognition strength regulates the choice between extrafollicular plasma cell and germinal center B cell differentiation. J Exp Med. 2006;203(4):1081-1091.

113. Quinn KM, et al. Antigen expression determines adenoviral vaccine potency independent of IFN and STING signaling. J Clin Invest. 2015;125(3):1129-1146.

114. Shaulov A, Murali-Krishna K. CD8 T cell expansion and memory differentiation are facilitated by simultaneous and sustained exposure to antigenic and inflammatory milieu. JImmunol. 2008;180(2):1131-1138.

115. Gupta RK, Chang AC, Griffin P, Rivera R, Siber GR. In vivo distribution of radioactivity in mice after injection of biodegradable polymer microspheres containing 14C-labeled tetanus toxoid. Vaccine. 1996;14(15):1412-1416.

116. Kool M, Fierens K, Lambrecht BN. Alum adjuvant: some of the tricks of the oldest adjuvant. JMed Microbiol. 2012;61(pt 7):927-934.

117. Hutchison S, Benson RA, Gibson VB, Pollock $\mathrm{AH}$, Garside P, Brewer JM. Antigen depot is not required for alum adjuvanticity. FASEB $J$. 2012;26(3):1272-1279.

118. Noe SM, Green MA, HogenEsch H, Hem SL. Mechanism of immunopotentiation by aluminum-containing adjuvants elucidated by the relationship between antigen retention at the inoculation site and the immune response. $\mathrm{Vac}$ cine. 2010;28(20):3588-3594.

119. Preis I, Langer RS. A single-step immunization by sustained antigen release. J Immunol Methods. 1979;28(1-2):193-197.

120. Singh M, Chakrapani A, O'Hagan D. Nanoparticles and microparticles as vaccine-delivery systems. Expert Rev Vaccines. 2007;6(5):797-808.

121. Jain S, O'Hagan DT, Singh M. The long-term potential of biodegradable poly(lactideco-glycolide) microparticles as the nextgeneration vaccine adjuvant. Expert Rev Vaccines. 2011;10(12):1731-1742.

122. Kim YC, Park JH, Prausnitz MR. Microneedles for drug and vaccine delivery. Adv Drug Deliv Rev. 2012;64(14):1547-1568.

123. Hirobe S, et al. Development and clinical study of a self-dissolving microneedle patch for transcutaneous immunization device. Pharm Res. 2013;30(10):2664-2674.

124.Sullivan SP, et al. Dissolving polymer microneedle patches for influenza vaccination. Nat Med. 2010;16(8):915-920. 
125. Tsioris K, Raja WK, Pritchard EM, Panilaitis B, Kaplan DL, Omenetto FG. Fabrication of silk microneedles for controlled-release drug delivery. Adv Funct Mater. 2012;22(2):330-335.

126. Demuth PC, Garcia-Beltran WF, Ai-Ling ML, Hammond PT, Irvine DJ. Composite dissolving microneedles for coordinated control of antigen and adjuvant delivery kinetics in transcutaneous vaccination. Adv Funct Mater. 2013;23(2):161-172.

127. Zaric M, et al. Dissolving microneedle delivery of nanoparticle-encapsulated antigen elicits efficient cross-priming and Th1 immune responses by murine Langerhans cells. J Invest Dermatol. 2015;135(2):425-434

128. DeMuth PC, Min Y, Irvine DJ, Hammond PT. Implantable silk composite microneedles for programmable vaccine release kinetics and enhanced immunogenicity in transcutaneous immunization. Adv Healthc Mater. 2014;3(1):47-58.

129. DeMuth PC, et al. Polymer multilayer tattooing for enhanced DNA vaccination. Nat Mater. 2013;12(4):367-376.

130. Zhang J, et al. Stabilization of vaccines and antibiotics in silk and eliminating the cold chain. Proc
Natl Acad Sci U S A. 2012;109(30):11981-11986. 131. Ali OA, Huebsch N, Cao L, Dranoff G, Mooney DJ. Infection-mimicking materials to program dendritic cells in situ. Nat Mater. 2009;8(2):151-158.

132. Ali OA, et al. Identification of immune factors regulating antitumor immunity using polymeric vaccines with multiple adjuvants. Cancer Res. 2014;74(6):1670-1681.

133. Ali OA, Emerich D, Dranoff G, Mooney DJ. In situ regulation of DC subsets and $\mathrm{T}$ cells mediates tumor regression in mice. Sci Transl Med. 2009;1(8):8ra19. 